\title{
Numerical Computation of Dufour and Soret Effects on Radiated Material on a Porous Stretching Surface with Temperature-Dependent Thermal Conductivity
}

\author{
Tahir Naseem ${ }^{1}\left(\mathbb{D}\right.$, Umar Nazir ${ }^{2}$, Essam R. El-Zahar ${ }^{3,4, *(\mathbb{D}, \text { Ahmed M. Algelany }}{ }^{3,5}$ and Muhammad Sohail $^{2} \mathbb{D}$ \\ 1 Department of Mathematics, Government Postgraduate College Haripur, P.O. Box 22620, \\ Haripur 22620, Pakistan; tahir.gch@gmail.com \\ 2 Department of Applied Mathematics \& Statistics, Institute of Space Technology, P.O. Box 2750, \\ Islamabad 44000, Pakistan; nazir_u2563@yahoo.com (U.N.); muhammad_sohail111@yahoo.com (M.S.) \\ 3 Department of Mathematics, College of Science and Humanities in Al-Kharj, \\ Prince Sattam Bin Abdulaziz University, P.O. Box 83, Al-Kharj 11942, Saudi Arabia; \\ ah.mohamed@psau.edu.sa \\ 4 Department of Basic Engineering Science, Faculty of Engineering, Menoufia University, \\ Shebin El-Kom 32511, Egypt \\ 5 Department of Mathematics, Faculty of Sciences, Fayoum University, Fayoum 63514, Egypt \\ * Correspondence: er.elzahar@psau.edu.sa
}

\section{check for} updates

Citation: Naseem, T.; Nazir, U.; El-Zahar, E.R.; Algelany, A.M.; Sohail, M. Numerical Computation of Dufour and Soret Effects on Radiated Material on a Porous Stretching Surface with Temperature-Dependen Thermal Conductivity. Fluids 2021, 6, 196. https://doi.org/10.3390/ fluids6060196

Academic Editor: Igor

V. Miroshnichenko

Received: 18 April 2021

Accepted: 18 May 2021

Published: 24 May 2021

Publisher's Note: MDPI stays neutral with regard to jurisdictional claims in published maps and institutional affiliations.

Copyright: (c) 2021 by the authors. Licensee MDPI, Basel, Switzerland. This article is an open access article distributed under the terms and conditions of the Creative Commons Attribution (CC BY) license (https:// creativecommons.org/licenses/by/ $4.0 /)$.

\begin{abstract}
The current research is prepared to address the transport phenomenon in a hydromagnetized flow model on a porous stretching sheet. Mass and heat transport are modeled via temperature dependent models of thermal conductivity and diffusion coefficients. Accordingly, the involvement of radiation, chemical reaction, the Dufour effect, and the Soret effect are involved. The flow presenting expression has been modeled via boundary layer approximation and the flow is produced due to the experimental stretching sheet. The governing equations have been approximated numerically via shooting method. The efficiency of the scheme is established by including the comparative study. Moreover, a decline in the velocity field is recorded against the escalating values of the porosity parameter and the magnetic parameter.
\end{abstract}

Keywords: porous medium; radiated heat flux; stretching sheet; nusselt number; boundary layer theory; shooting method

\section{Introduction}

Recently, researchers have paid great attention to the investigation of the compartment of thermal and species transportation in many engineering processes. For instance, Sohail et al. [1] modeled the phenomenon of thermal and mass transportation with variable thermal conduction and diffusion coefficient in an Oldroyd-B fluid model. They used the boundary layer approach to model the physical happening on a stretched surface. Group similarity analysis was used to transfigure the coupled system of modeled laws into ordinary differential equations. They handled the transfiguring model via the optimal homotopy method. They presented the error analysis and comparative investigation. They reported that higher values of the Prandtl number results in a decline in the thermal profile. Khan et al. [2] modeled the rheology of the Powell-Eyring model in cylindrical coordinates. They used the involvement of the magnetohydrodynamic (MHD) effect in momentum distribution and a variable model of thermal conductivity in temperature profile. Sohail et al. [3] studied the species and thermal transportation in a couple stress model flowing over a stretched surface. They considered the variable magnetic field in the momentum equation, depending upon the spatial variables. The phenomenon of thermal transport is discussed under temperature-dependent thermal conductivity and radiation energy in their exploration, and they also discussed their entropy analysis. They 
used the OHAM tool to approximate the solution of coupled nonlinear flow presenting equation. An error analysis is computed to show the authenticity of the applied scheme. Khan et al. [4] studied the compartment of Joule heating, viscous dissipation, radiation, and chemical reaction for the flow of micropolar fluid over a stretching surface obeying convective thermal conditions. They presented the graphical convergence analysis through h-curves. Hayat et al. [5] developed the modeling of cross fluid over a stretching sheet in the presence of homogeneous-heterogeneous reactions. They found that higher values of the Weissenberg parameter retard the velocity profile. Sohail et al. [6] investigated the mechanism of entropy in the Casson model under variable properties. They addressed the analytical solution of the developed problem. They mentioned that augmenting the values of the fluid parameter is responsible for the control of the turbulence in fluid motion. Shah et al. [7] studied the hybrid nanofluid in porous annulus and explored the phenomenon of entropy generation by augmenting the Bejan number numerically via a finite volume scheme. In another inspection, thermal transport with a modified heat flux model in micropolar fluid is scrutinized. They used the HAM procedure to investigate the features of various flow profiles. They mentioned that the escalating values of the magnetic parameter and the Prandtl number enhance the heat transmission rate, while the Eckert number shows the reverse. The involvement of Joule heating in water-based silver and copper nanoparticles was examined by Shah et al. [8]. They studied the flow features in a porous medium. The investigation of entropy was reported by them. They reported that the escalating values of the rotation parameter increased the velocity field. Moreover, it is examined that the inertial coefficient and the porosity parameter decline the radial velocity component. Khan et al. [9] studied the radiated blood flow in a slippery sheet by incorporating the heat generation/absorption contribution. They modeled the fluid presenting expressions in a curved, stretched surface. They solved the modeled equations via the bvp4c procedure. The involvement of modified heat flux is incorporated by them to study the momentum and thermal transport. They considered the exponentially stretching sheet over which flow is produced. Their error analysis is presented to show the convergence of method. They found that higher values of porosity parameter retard the velocity and augmentation in the thermal profile. Rasool and Shafique studied the aspects of heat and mass transfer in the Powell-Earing model with convective boundary conditions and the radiation phenomenon. They found a decline in concentration profile against the Schmidt number. The modeling of Casson fluid with heat and mass transportation over a nonlinear stretching sheet was numerically investigated by Rasool et al. [10]. They found that higher estimations in the slip parameter retards the velocity, thermal, and concentration fields. In addition, they noticed the incrimination in the temperature field against the Eckert number. Al-Khaled and Khan [11] examined the phenomenon of gyrotactic microorganisms with heat and mass transportation in the Casson model. They used the HAM approach to approximate the solutions of coupled transformed boundary layer equations. They observed the augmentation in temperature profile against the large values of the radiation parameter. Furthermore, they recorded the decline in the microorganisms' fields against the Lewis and Péclet numbers. In [12], Mondal and Mukhopadhyay scrutinized the thermal transport in Newtonian fluid passing over an exponential porous sheet. They tackled the transformed ODEs numerically and recorded the decline in temperature field against the Prandtl number. Khan et al. [13] carried out the analysis of heat transfer over an unsteady porous stretching sheet for the viscoelastic model. Alam and Marwat [14] studied the mass and heat transport in stagnation point fluids over variable thickness porous sheets. They solved the resulting equations numerically and discussed the cases of suction and blowing. Some important aspects are mentioned in [15-18] and references therein.

The available literature reveals that no attempt has been made to explore the compartment of variable thermal conductivity and diffusion coefficient with the Soret and Dufour effects as magnetic nanofluid passes over an exponentially stretching sheet. This exploration fills the gap in the research, and will be used as a founding tool for the other researchers to contribute their input to open literature. This paper is organized as follows: 
Section 1 contains the literature survey, modeling is included in Section 2, Section 3 contains the methodology, results are explained in Section 4, and the key findings are reported in Section 5 .

\section{Governing Equations}

The coordinate system is considered in two dimensions such that the $x$-axis is directed to the stretching sheet inserted inside the porous medium, and the $y$-axis moves upward from the sheet as shown in Figure 1. The stretched and free stream velocities are defined as

$$
u_{w}(x)=a e^{\frac{x}{l}}, u_{\infty}(x)=0,
$$

where $x, l$, and $a>0$ are coordinates parallel to the sheet, the length of the stretching sheet, and the constant, respectively. A nonuniform, transversely applied magnetic field of strength $B=B_{o} e^{x / l}$ is applied, as mentioned in Figure 1. Furthermore, it is assumed that no external electric field exists, therefore the impact of polarization of charges is negligibly small. The induced magnetic field created by the nanofluid (electrically conducting) motion is also in negligibly small amounts in comparison with the applied field. The governing equations in usual dimensional forms are:

$$
\begin{gathered}
\frac{\partial u}{\partial x}+\frac{\partial v}{\partial y}=0 \\
u \frac{\partial u}{\partial x}+v \frac{\partial u}{\partial y}-\frac{\mu}{\rho} \frac{\partial^{2} u}{\partial y^{2}}+\left(\frac{v}{K}+\frac{\sigma B_{0}^{2}}{\rho}\right) u=0 \\
u \frac{\partial C}{\partial x}+v \frac{\partial C}{\partial y}-\frac{\partial}{\partial y}\left[D(T) \frac{\partial C}{\partial y}\right]+\Gamma\left(C-C_{0}\right)-\frac{\partial^{2} T}{\partial y^{2}} \frac{D_{T} k_{T}}{T_{\infty}}=0 \\
u \frac{\partial T}{\partial x}+v \frac{\partial T}{\partial y}-\frac{1}{\rho C p} \frac{\partial}{\partial y}\left[K(T) \frac{\partial T}{\partial y}\right]+\frac{1}{\rho C p} \frac{\partial Q_{r}}{\partial y}-\tau\left[\left(\frac{\partial T}{\partial y}\right)^{2} \frac{D_{T}}{T_{\infty}}+D_{B} \frac{\partial T}{\partial y} \frac{\partial C}{\partial y}\right]-\frac{D_{T} k_{T}}{C_{s} C_{p^{*}}} \frac{\partial^{2} C}{\partial y^{2}}=0 .
\end{gathered}
$$

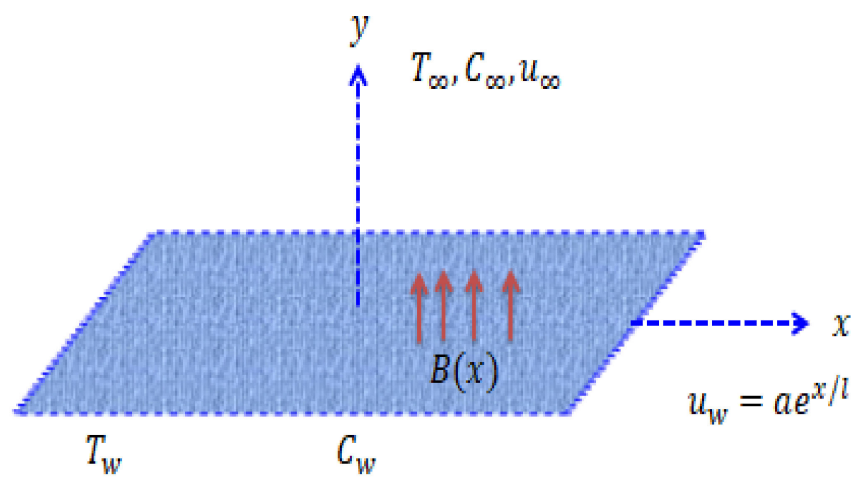

Figure 1. Flow diagram with coordinates. one has:

Employing the Roseland approximation (radiation flux) for an optically thick layer

$$
Q_{r}=\frac{4 \sigma^{*}}{3 k^{*}} \frac{\partial T^{4}}{\partial y} .
$$

The term $T^{4}$ in radiation flux can be solved by the Taylor Series about $T_{\infty}$ and, neglecting higher terms, we have:

$$
T^{4}=4 T_{\infty}^{3} T-3 T_{\infty}^{4}
$$


The subjected boundary conditions are:

$$
\begin{gathered}
u=u_{w}(\mathrm{x})=\mathrm{ae}^{x / l}, v=0, C=C_{w}(x), T=T_{w}(x) \text { at } y=0 \\
u \rightarrow 0, v \rightarrow 0, C \rightarrow C_{\infty}, T \rightarrow T_{\infty}=n T_{w}(x)+(1-n) T_{0}, \text { as } y \rightarrow \infty,
\end{gathered}
$$

where the constant thermal stratification parameter $n=\frac{m_{1}}{1+m_{1}}, m_{1}$ constant can be taken in many ways as $0 \leq n<1$. The prescribed temperature and concentration over the stretchable surface can be defined as

$$
T_{w}(x)=T_{\infty}+T_{0} \mathrm{e}^{\frac{x}{l}}, C_{w}(x)=C_{\infty}+C_{0} \mathrm{e}^{x / l},
$$

where $T_{0}$ and $C_{0}$ are the reference temperature and the reference concentration, respectively. An appropriate stream function $\psi=\psi(x, y)$ satisfyies the continuity of Equation (1), so that the flow equations converted to nonlinear ordinary differential equations, with the similarity transformations are taken as

$$
\begin{gathered}
\psi=\sqrt{2 l a} \mathrm{e}^{\frac{x}{T}} f(\eta), \eta=y \sqrt{\frac{a}{2 v l}} \mathrm{e}^{\frac{x}{T}}, u=\frac{\partial \psi}{\partial y}, v=-\frac{\partial \psi}{\partial x}, \\
\theta(\eta)\left(-T_{\infty}+T_{w}\right)=\left(-T_{\infty}+T\right), \varnothing(\eta)\left(-C_{\infty}+C_{w}\right)=\left(-C_{\infty}+C\right) .
\end{gathered}
$$

After applying the above transformation, Equations (2)-(4) become

$$
\begin{gathered}
f^{\prime \prime \prime}(\eta)-(k 1+M) f^{\prime}(\eta)+f(\eta) f^{\prime \prime}(\eta)-2(f \prime)^{2}(\eta)=0, \\
\left(1+\delta_{1} \theta(\eta)\right) \phi^{\prime \prime}(\eta)-L e f \prime(\eta) \phi(\eta)+L e f(\eta) \phi \prime(\eta)-L e \Gamma \phi(\eta)+\operatorname{SrLe} \theta^{\prime \prime}(\eta)=0 \\
\frac{1}{\operatorname{Pr}}\left(1+R+\delta_{2} \theta(\eta)\right) \theta^{\prime \prime}(\eta)+N t\left(\theta^{\prime}\right)^{2}(\eta)+\operatorname{Dr}^{\prime \prime}(\eta)+f(\eta) \theta \prime(\eta)-f \prime(\eta) \theta(\eta)-\left(\frac{n}{1-n}\right) f_{\prime}(\eta)+N b \theta \prime(\eta) \phi \prime(\eta)=0
\end{gathered}
$$

The corresponding boundary conditions (7) become

$$
\begin{gathered}
f(\eta)=0, f^{\prime}(\eta)=1, \phi(\eta)=1, \theta(\eta)=1 \text { at } \eta=0, \\
f^{\prime}(\eta) \rightarrow 0, \phi(\eta) \rightarrow 0, \theta(\eta) \rightarrow 0 \text { at } \eta \rightarrow \infty .
\end{gathered}
$$

The skin friction, Sherwood number, and Nusselt number for the current study are regarded as

$$
\begin{gathered}
N u=\frac{x Q_{W}}{K(T)\left(T_{w}-T_{\infty}\right)}, S h=\frac{x Q_{m}}{D_{B}(T)\left(C_{w}-C_{\infty}\right)}, C_{F}=\frac{\tau_{W}}{u_{w}^{2} \rho_{f}}, \\
\text { where } Q_{W}=-K(T) \frac{\partial T}{\partial y}, Q_{m}=-D_{B}(T) \frac{\partial C}{\partial y}, \tau_{W}=\mu \frac{\partial u}{\partial y} a t y=0 .
\end{gathered}
$$

The related terms of dimensionless reduced Nusselt number $-\theta \prime(0)$, reduced Sherwood number $-\phi^{\prime}(0)$, and skin friction coefficient $C f x$ are

$$
\begin{gathered}
C_{F X}=C f \sqrt{\left(\frac{2 l}{x}\right) R e_{x},-}(1+R) \theta^{\prime}(0)=\frac{N u}{\left(1+\frac{4}{3}\right) \sqrt{\frac{x}{2 l} R e_{x}}}, \\
-\phi^{\prime}(0)=\frac{S h}{\sqrt{\left(\frac{x}{2 l}\right) R e_{x}}},
\end{gathered}
$$

where $R e_{x}=\frac{u_{w}(\mathrm{x})}{y}$ is the local Reynolds number. In order to get a numerical solution of the developed equations, a numerical method is used based on shooting method.

The involved variables in the above equations are presented with the help of nomenclature as mentioned in the following section.

\section{Numerical Method for Solution}

With the use of similarity transformation, the nonlinear partial differential equations with subjected boundary conditions are converted to ordinary differential equations then solved by using the Shooting method. The methodology flow chart is shown in Figure 2. Here, the third-order nonlinear ordinary differential Equation (10) and second-order nonlin- 
ear ordinary differential Equations (11) and (12) have been reduced to first-order differential equations as follows

$$
\begin{gathered}
f=y_{1}, f^{\prime}=y_{2}, f^{\prime \prime}=y_{3}, f^{\prime \prime \prime}=(y y)_{1}, \\
(y y)_{1}=(k 1+M) y_{2}-y_{1} y_{3}+2\left(y_{2}\right)^{2}, \\
(y y)_{2}=\frac{1}{\frac{1}{P r}\left(1+R+\epsilon_{1} y_{7}\right)}\left\{-(N t)\left(y_{5}\right)^{2}-(D r)(y y)_{3}-y_{1} y_{5}+y_{2} y_{4}+\left(\frac{n}{1-n}\right) y_{2}-(N b) y_{5} y_{7}\right\}, \\
\phi=y_{6}, \phi^{\prime}=y_{7},(y y)_{3}, \\
\phi^{\prime \prime}=\frac{1}{\left(1+\epsilon_{2} y_{4}\right)}\left\{(L e) y_{2} y_{6}-(L e) y_{1} y_{7}+(L e)(\Gamma) y_{6}-(S r)(L e)(y y)_{2}\right\},
\end{gathered}
$$

The corresponding boundary condition becomes

$$
y_{1}(0)=0, y_{2}(0)=1, y_{4}(0)=0, y_{6}(0)=1, y_{2}(\infty)=0, y_{4}(\infty)=0, y_{6}(\infty)=0 \text {. }
$$

The iterative process will be terminated until the desired degree of accuracy $\left(10^{-7}\right)$ is obtained.
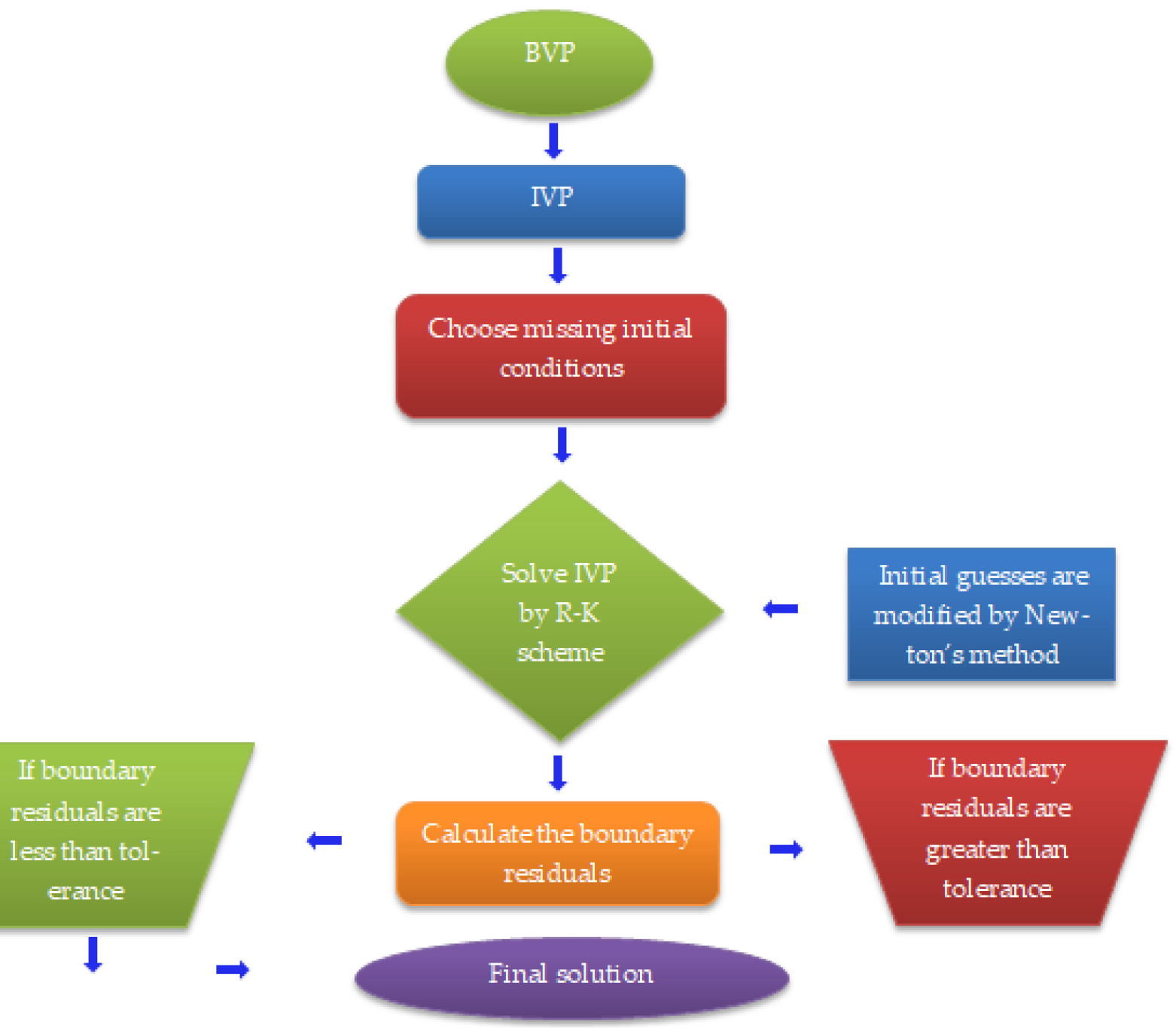

Figure 2. Flow chart of solution approach. 


\section{Results and Discussion}

The 2D mathematical model is designed with the help of transportation of mass diffusion, thermal energy, and momentum equations in the presence of constant magnetic and thermal radiation, including the role of the Dufour and Soret numbers using variable properties. Furthermore, the simulations and recorded outcomes are done in view of the graphs and tables via shooting scheme. The graphical simulations of various physical parameters on the TBL (thermal boundary layer) and the MBL (momentum boundary layer) are captured. The desired outcomes regarding motion of particles, heat energy, and diffusion of mass are captured below.

Flow of fluid particles versus physical parameters: The related outcomes of velocity against the variation of the porosity number $(k 1)$ and magnetic field $(M)$ are captured by Figures 3 and 4 . The involvement of $(k 1)$ on the motion of fluid particles are estimated by Figure 3. It is demonstrated in Figure 3 that flow slows down when $k 1$ is enhanced. The flow of fluid particles and the thickness in boundary layer of fluid at surface of the sheet decline versus large values of $k 1$. It is also estimated that $k 1$ generates the resistance during the flow of fluid particles and that this resistance results in a decreasing in motion of particles. Figure 4 captures the effect of $(M)$ on the velocity. In this figure, the motion in fluid particles becomes slower versus large values of $(M)$. This decreasing result in velocity because of the strength of $M$ generates drag force in flow called the Lorentz force. This force is plays an opposite role against the motion of particles when a constant magnetic field is taken along normal direction of flow over a stretched surface.

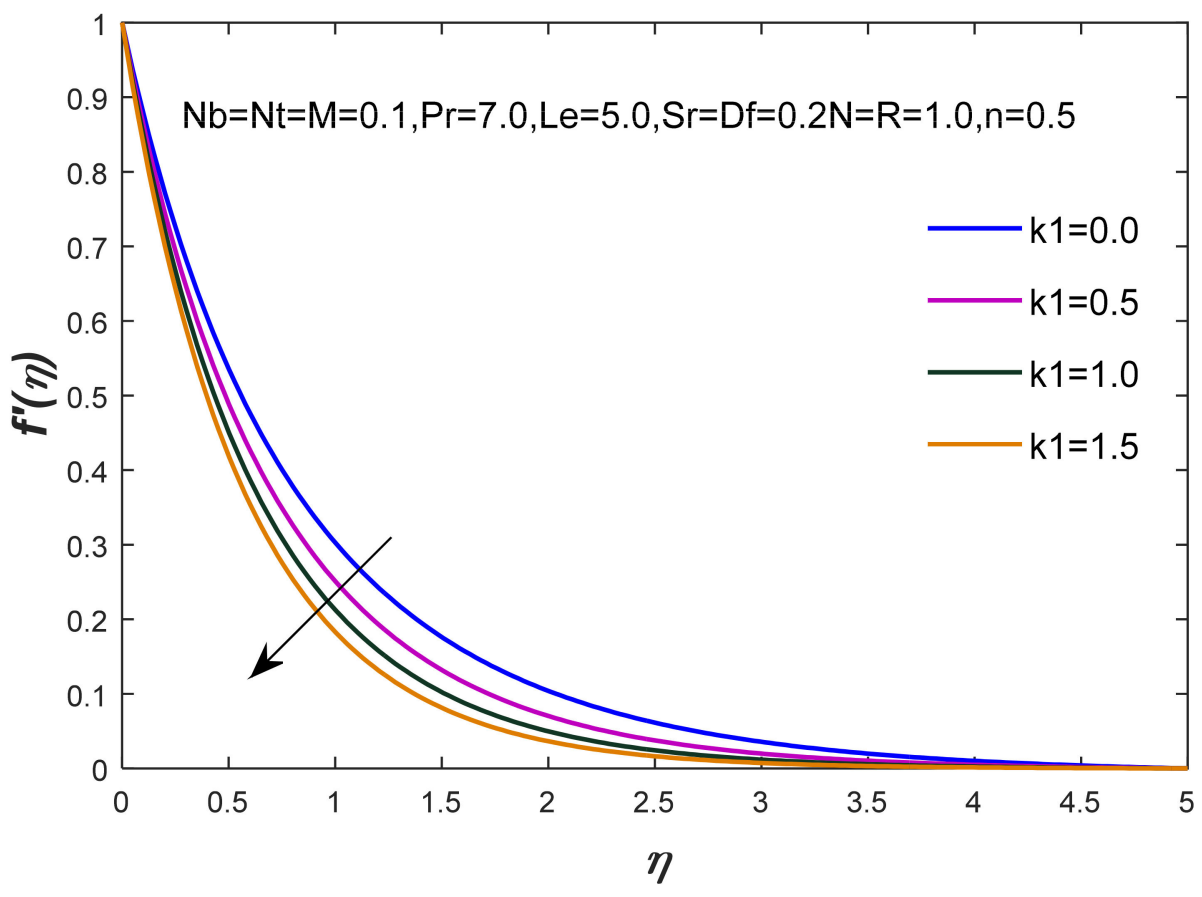

Figure 3. The impacts of $k 1$ on $f(\eta)$. 


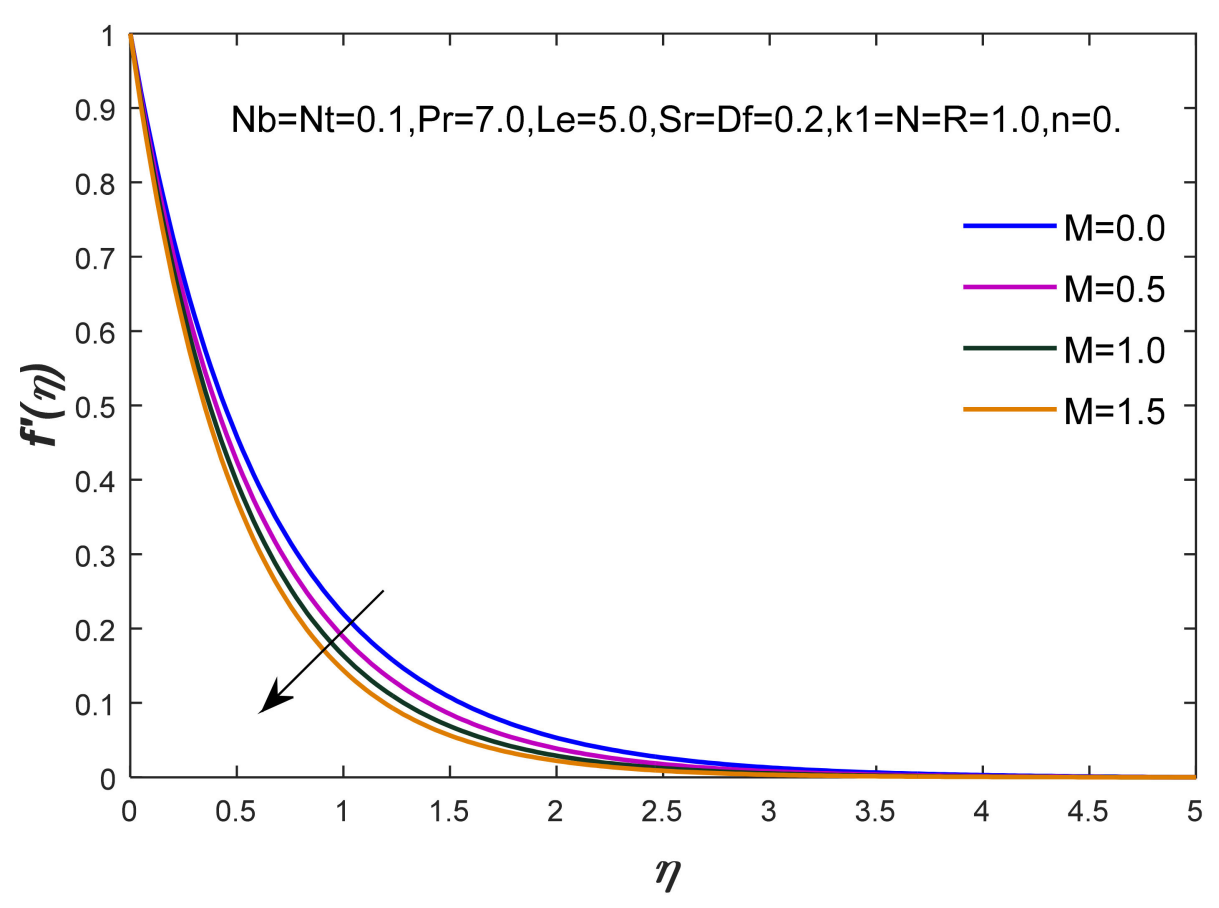

Figure 4. The impacts of $M$ on $f(\eta)$.

Temperature of fluid particles versus physical parameters: The noted observations of the stratification $(n)$, thermal radiation $(R)$, Prandtl $(\operatorname{Pr})$, magnetic field $(M)$, Dufour $(D r)$, thermophoresis $(\mathrm{Nt})$, Brownian motion $(\mathrm{Nb})$, thermal relaxation time $\left(\delta_{1}\right)$, and Soret $(\mathrm{Sr})$ numbers on the thermal boundary layer are estimated by Figures $5-12$. The temperature of fluid particles versus $(n)$ is computed in Figure 5. The improvement of thermal energy is conducted through the values of $n=0.0,0.1,0.15$, and 2 . The thickness of the thermal boundary layer is enhanced by using large values of $n$. Figure 6 characterizes the impact of $(R)$ on thermal energy. The heat energy is increased against enhancement in $(R)$, while an enhancement in $(R)$ generates more thermal performance in temperature regarding working fluid. Furthermore, the motion of fluid particles in view of an injected manner is greatly influenced by $(R)$. The performance of heat energy with respect to $(P r)$ is expressed in Figure 7. From this figure, it is observed that temperature declines due to large values of $(P r)$. The increase in $(P r)$ brings the reduction in the layers of the thermal boundary because $(P r)$ is based on momentum as well as thermal boundary layers. Hence, an increase in $(P r)$ leads to the reduction in performance of thermal energy. 


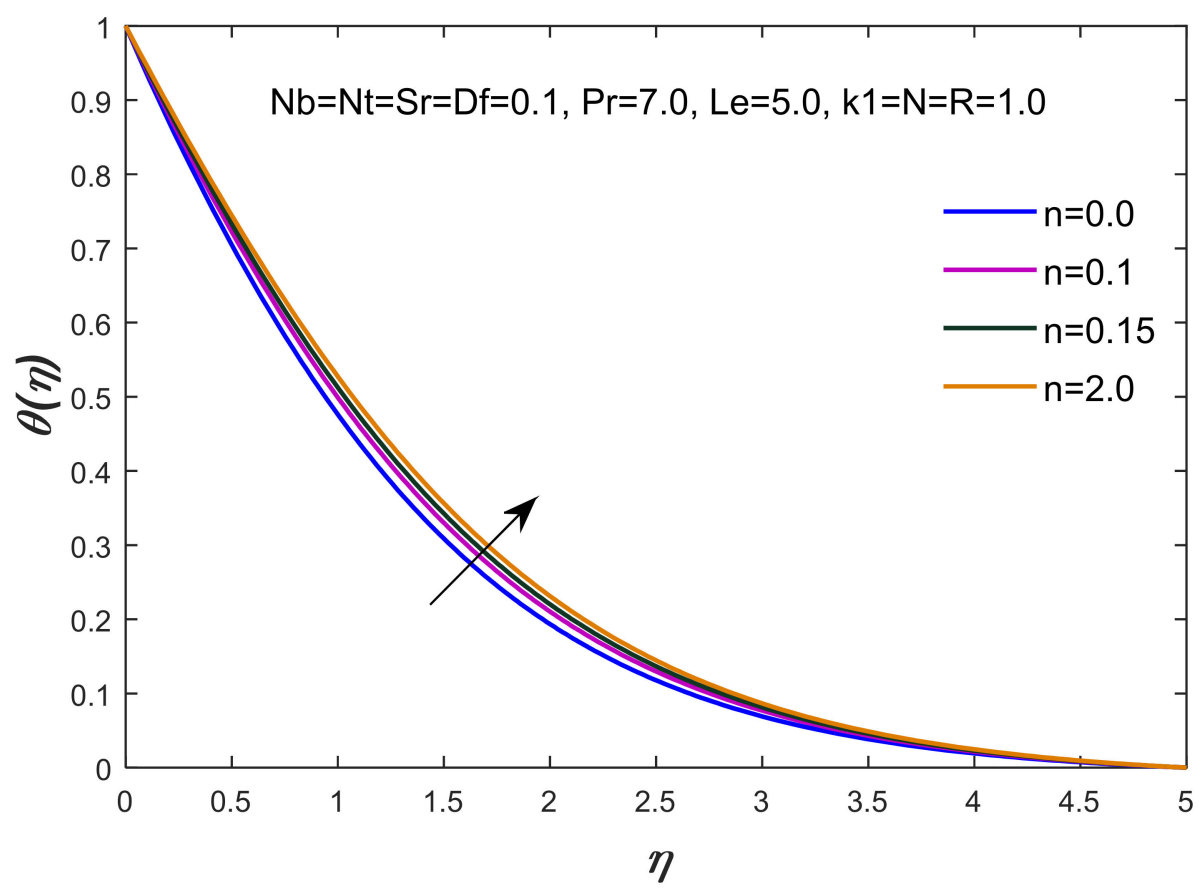

Figure 5. The impacts of $n$ on $\theta(\eta)$.

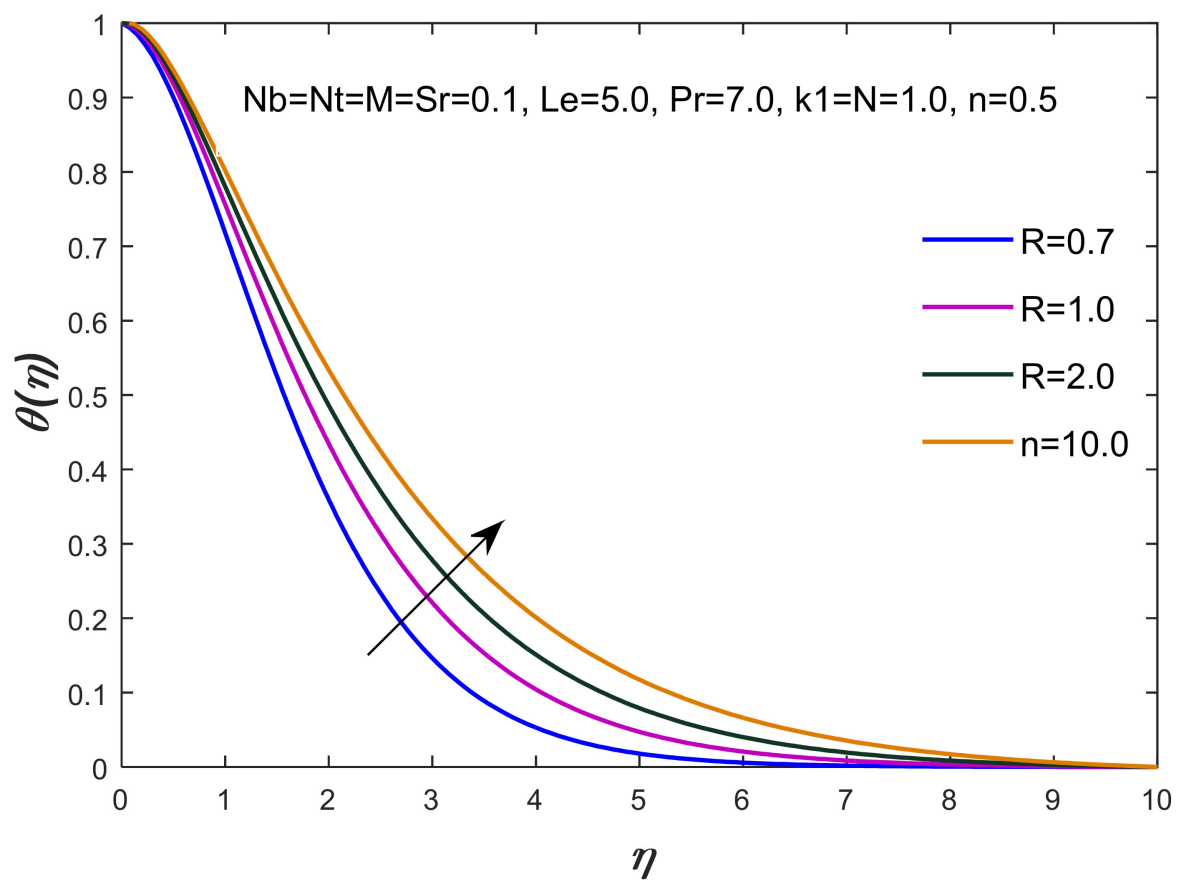

Figure 6. The impacts of $R$ on $\theta(\eta)$. 


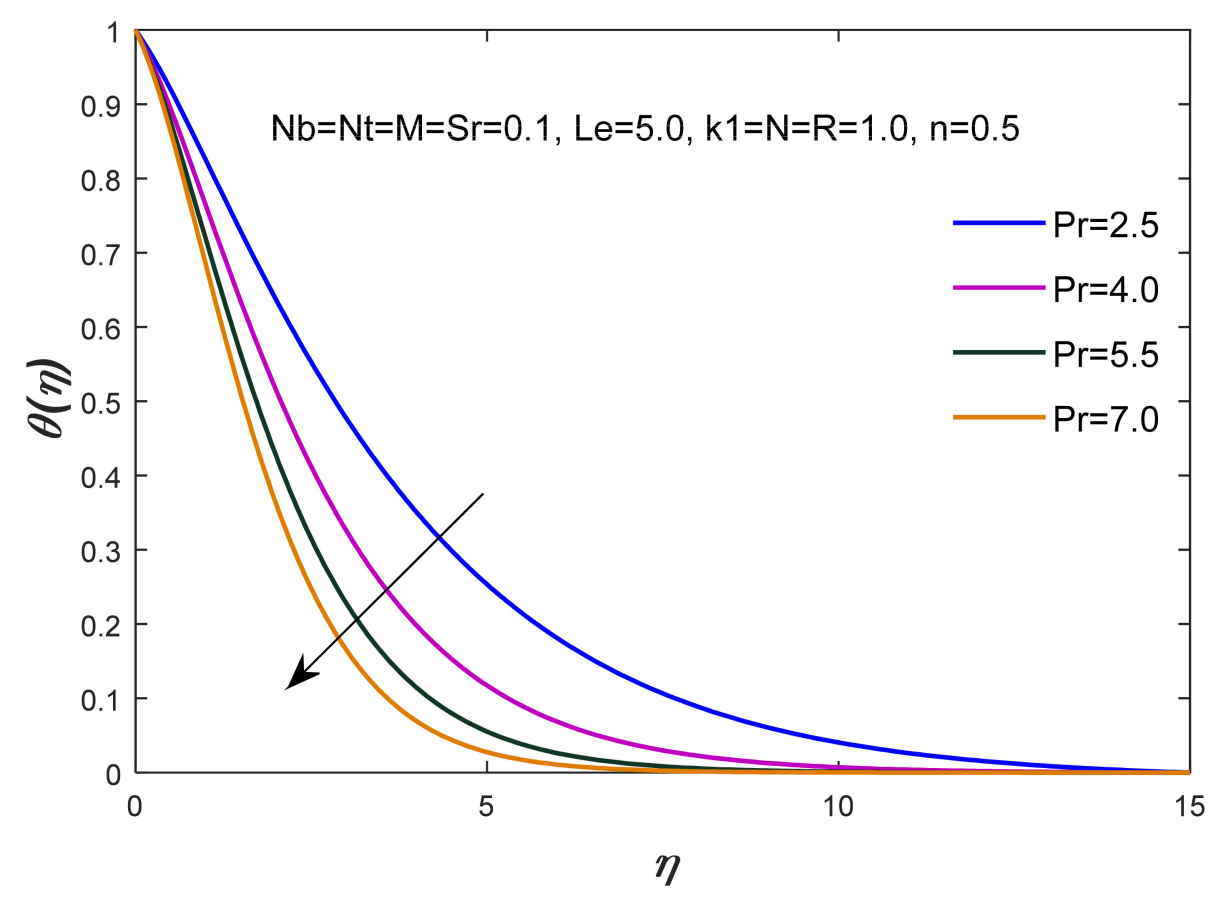

Figure 7. The impacts of $\operatorname{Pr}$ on $\theta(\eta)$.

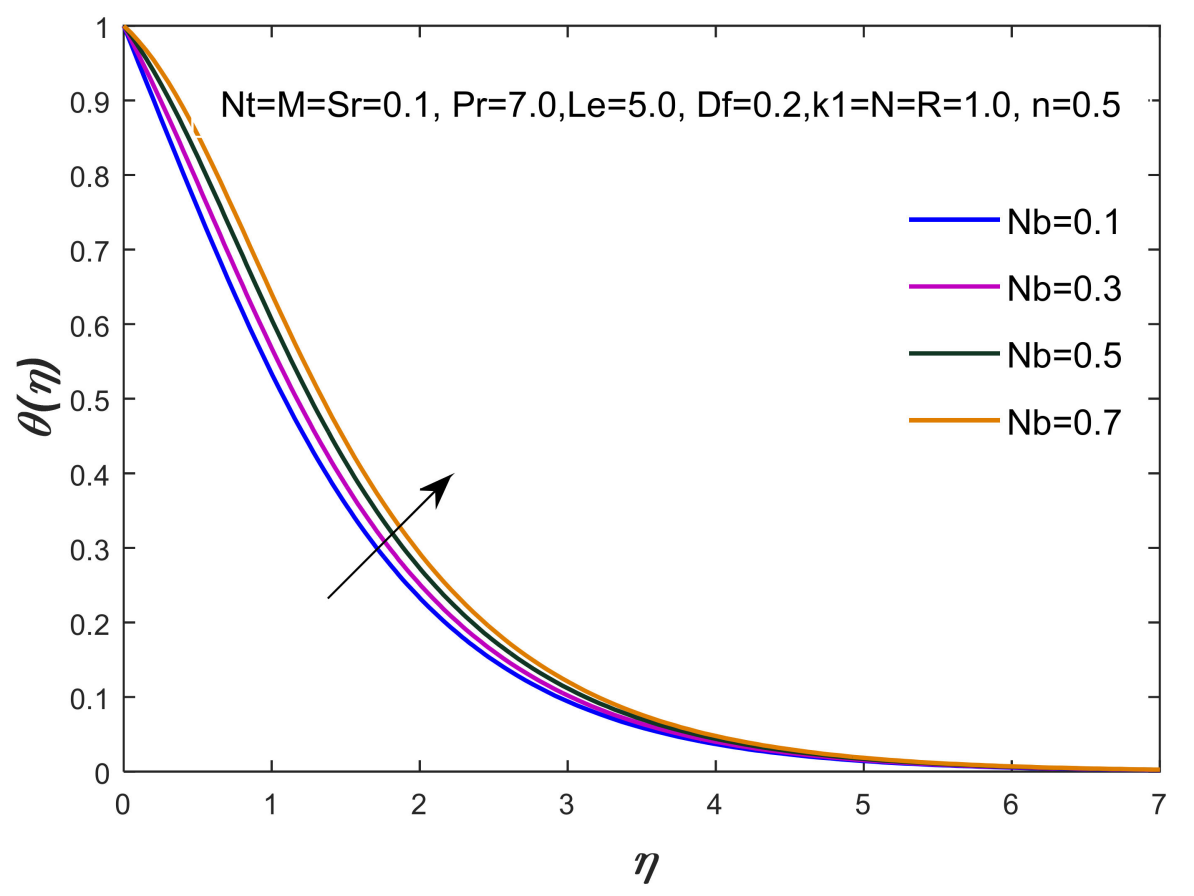

Figure 8. The impacts of $N b$ on $\theta(\eta)$. 


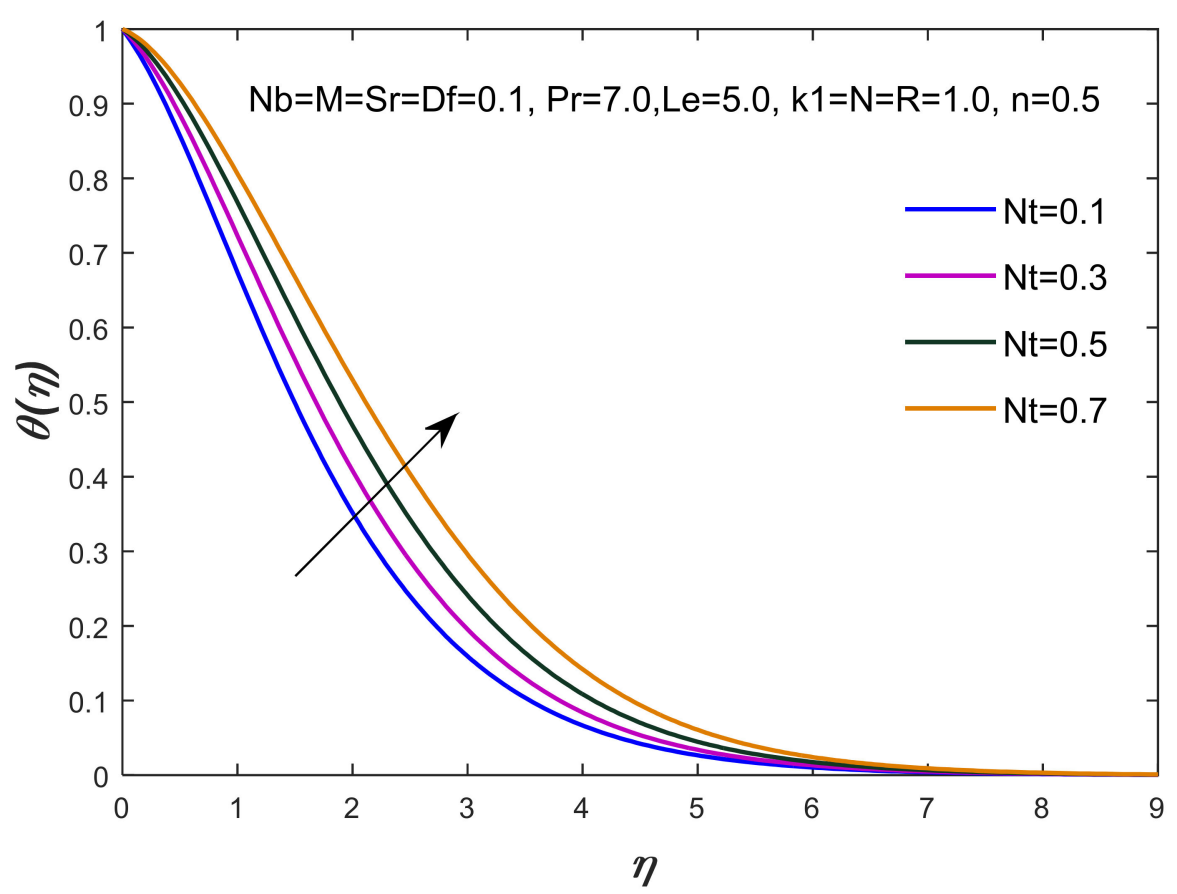

Figure 9. The impacts of $N t$ on $\theta(\eta)$.

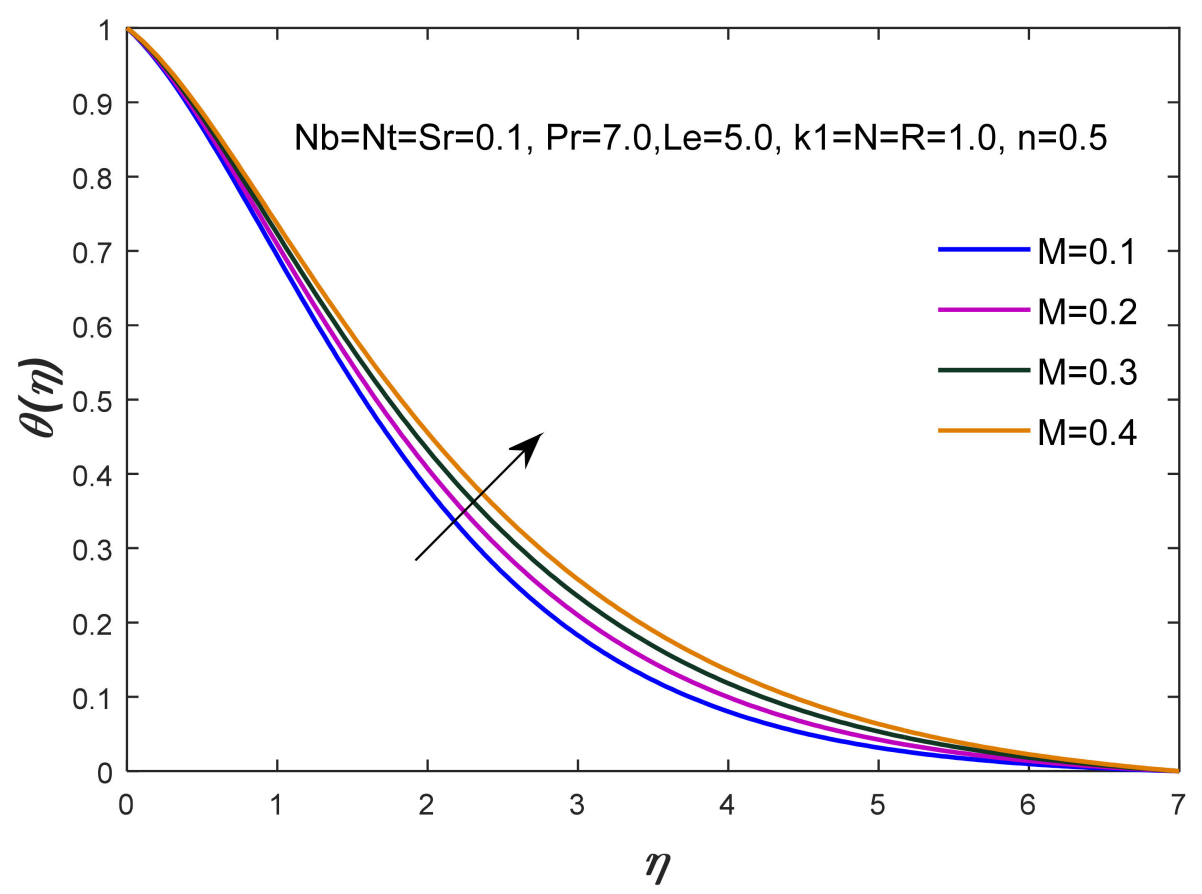

Figure 10. The impacts of $M$ on $\theta(\eta)$. 


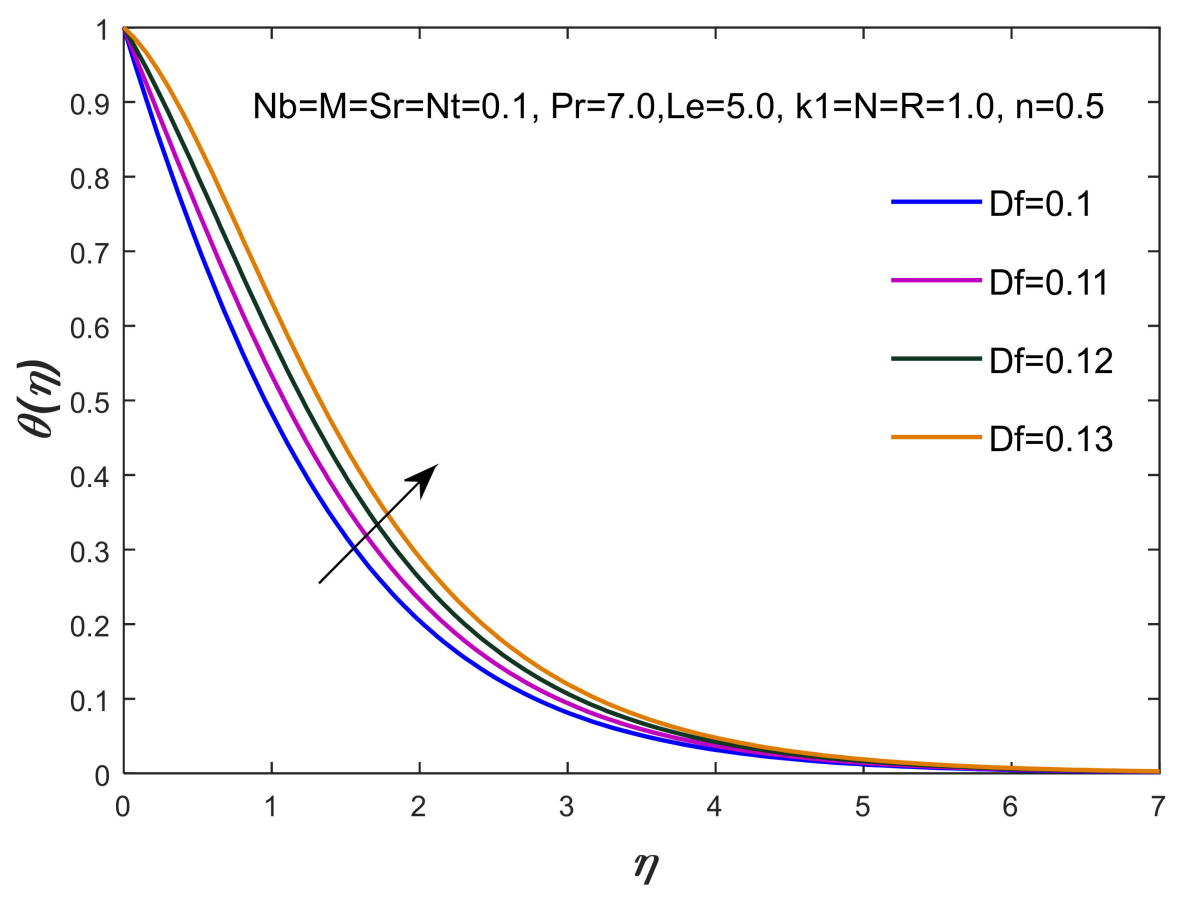

Figure 11. The impacts of $D f$ on $\theta(\eta)$.

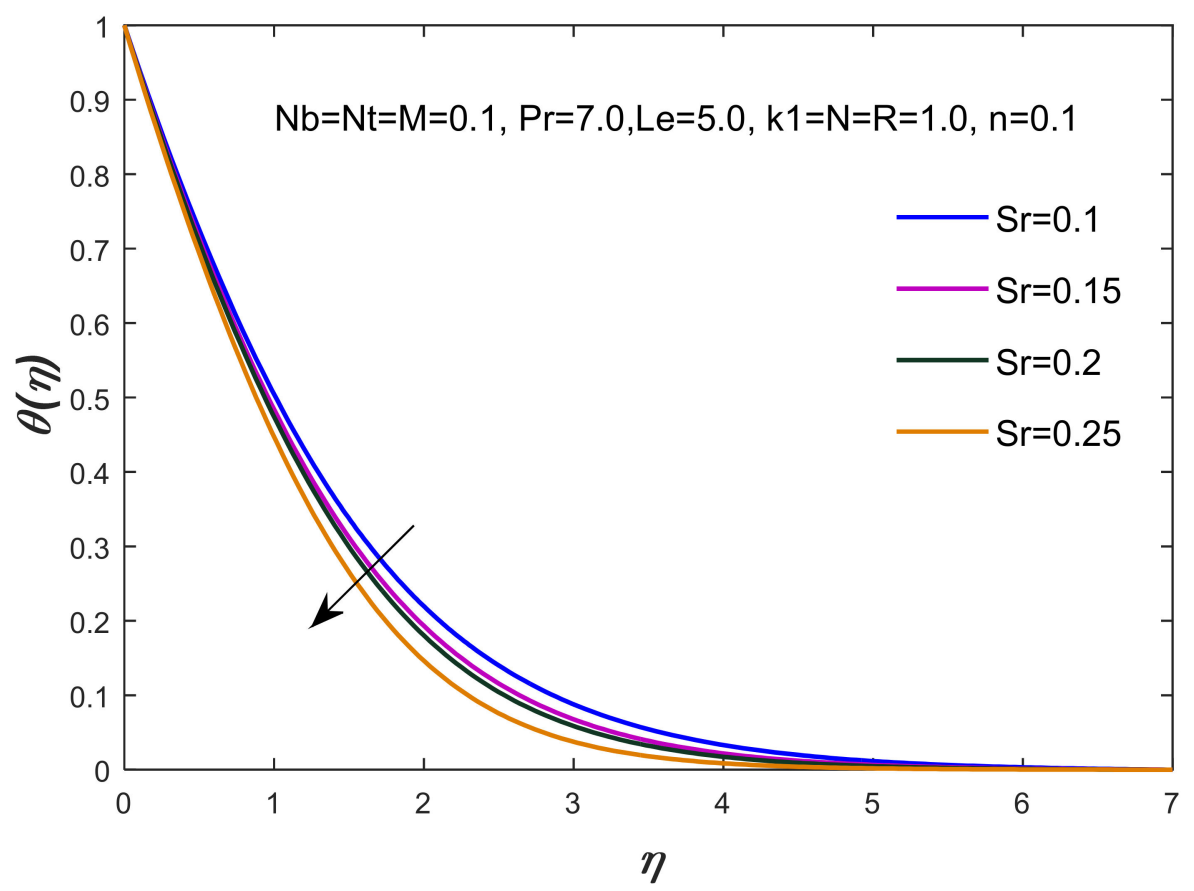

Figure 12. The impacts of $S r$ on $\theta(\eta)$.

Figures 8 and 9 demonstrate the character of $N b$ and $N t$ on the curves of heat energy. It is captured that, from these figures, the maximum production regarding thermal energy is gained by increasing the values of $N b$ and $N t$, while large values of $N b$ and $N t$ results in the reduction in the layers regarding the thermal boundary. The reason for this is generated in case of $N t$ due to stronger thermophoretic force in view of fluid particles, while this force is opposite of an imposed gradient of temperature. Hence, the performance of thermal energy is improved. 
The process of heat energy associated with a magnetic number on the profile of heat energy is demonstrated in Figure 10. The profile in view of thermal energy is enhanced versus large values of the magnetic number because of Lorentz force. The Lorentz force is that force which makes thin behavior regarding fluid particles. Therefore, the thinning behavior of fluids is experiences more thermal energy when using large values of $M$.

The dimensionless thermal energy related to various values of the Dufour number is demonstrated in Figure 11. In Figure 11, the distinct enhancement in thermal energy trough TBL is observed near the wall of the surface. Furthermore, a similar trend is noted on temperature curves versus various values of the Soret number (Figure 12).

Diffusion of fluid particles versus physical parameters: The simulations in view of concentration curves versus the variation in the thermal radiation $(R)$, Brownian motion $(N b)$, thermophoresis $(N t)$, magnetic field $(M)$, Lewis $(L e)$, and concentration relaxation time $\left(\delta_{2}\right)$ numbers are shown in Figures 13-19. The outcome of thermal energy versus thermal radiation number is simulated in Figure 13. The increment in $(R)$ is responsible for better performance in thermal energy.

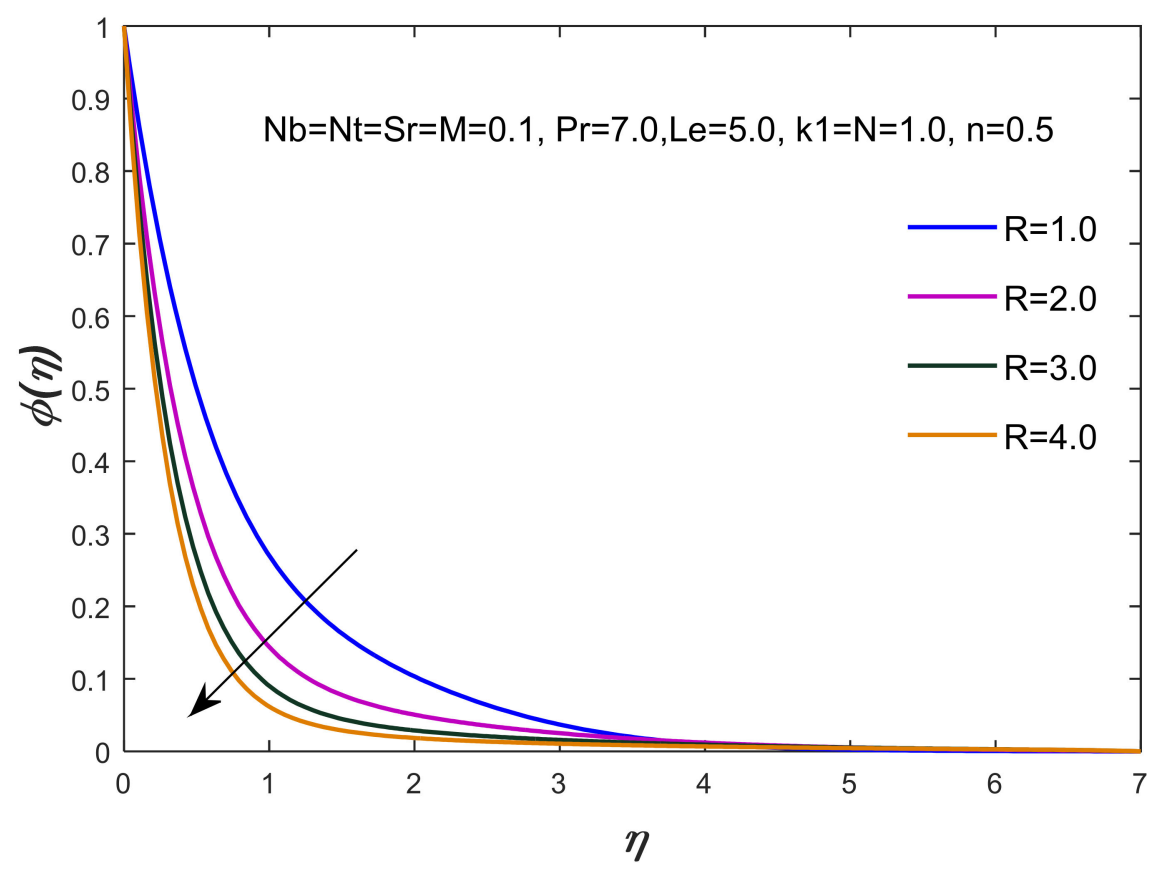

Figure 13. The impacts of $R$ on $\phi(\eta)$. 


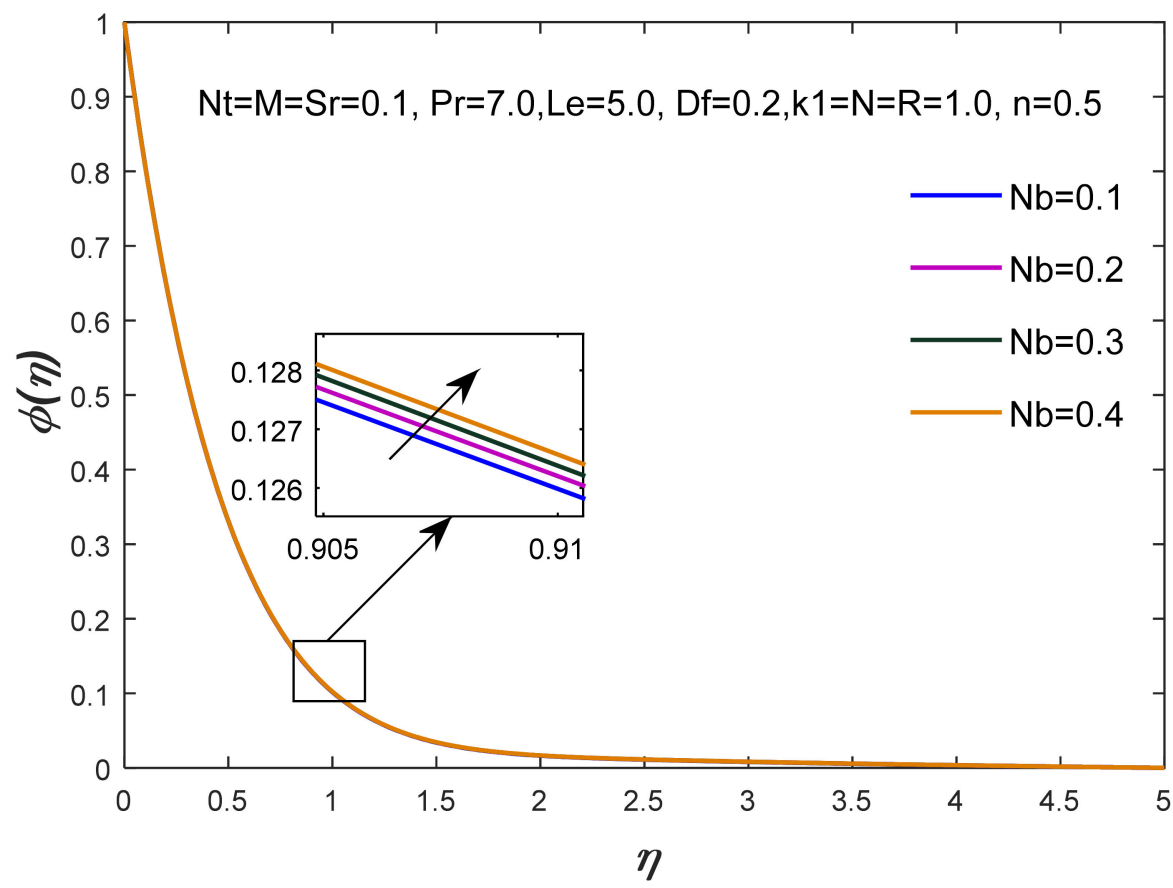

Figure 14. The impacts of $N b$ on $\phi(\eta)$.

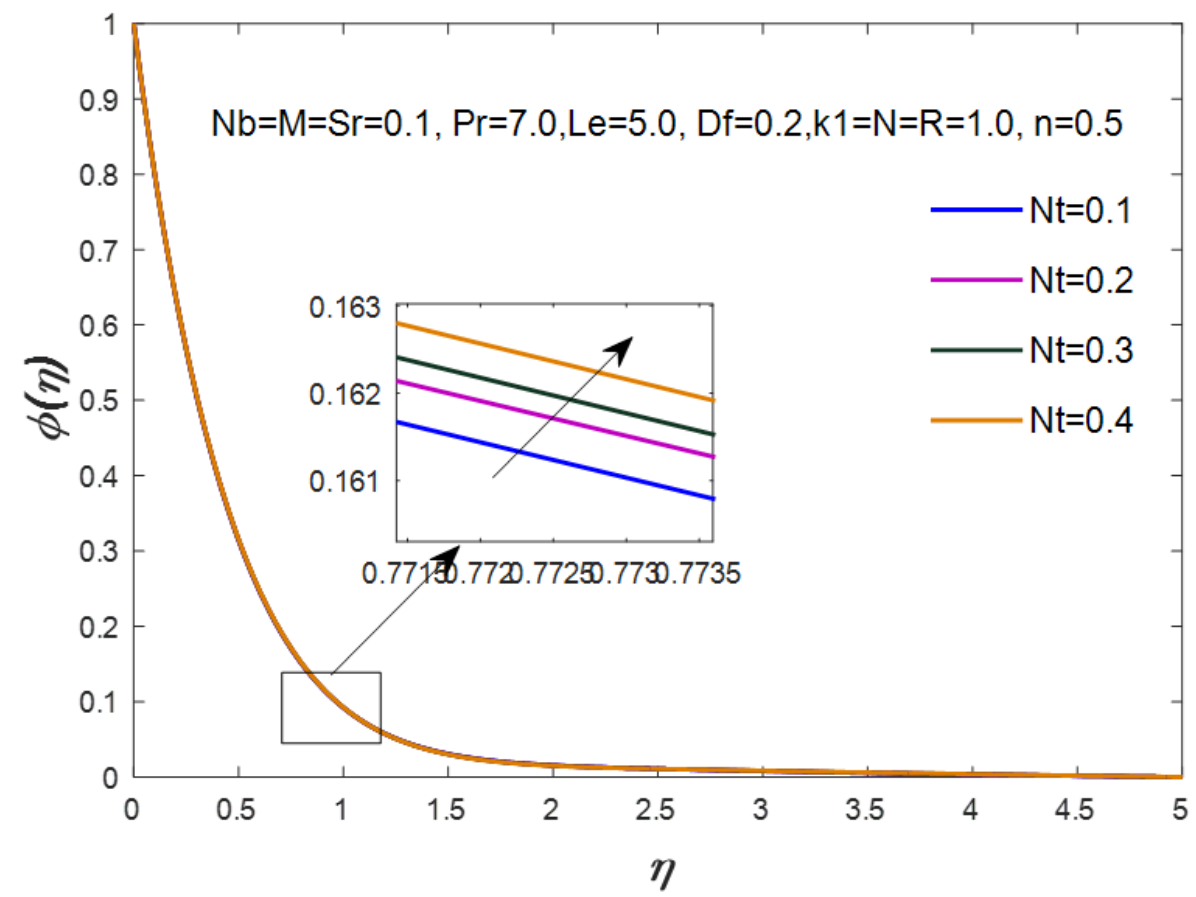

Figure 15. The impacts of $N t$ on $\phi(\eta)$. 


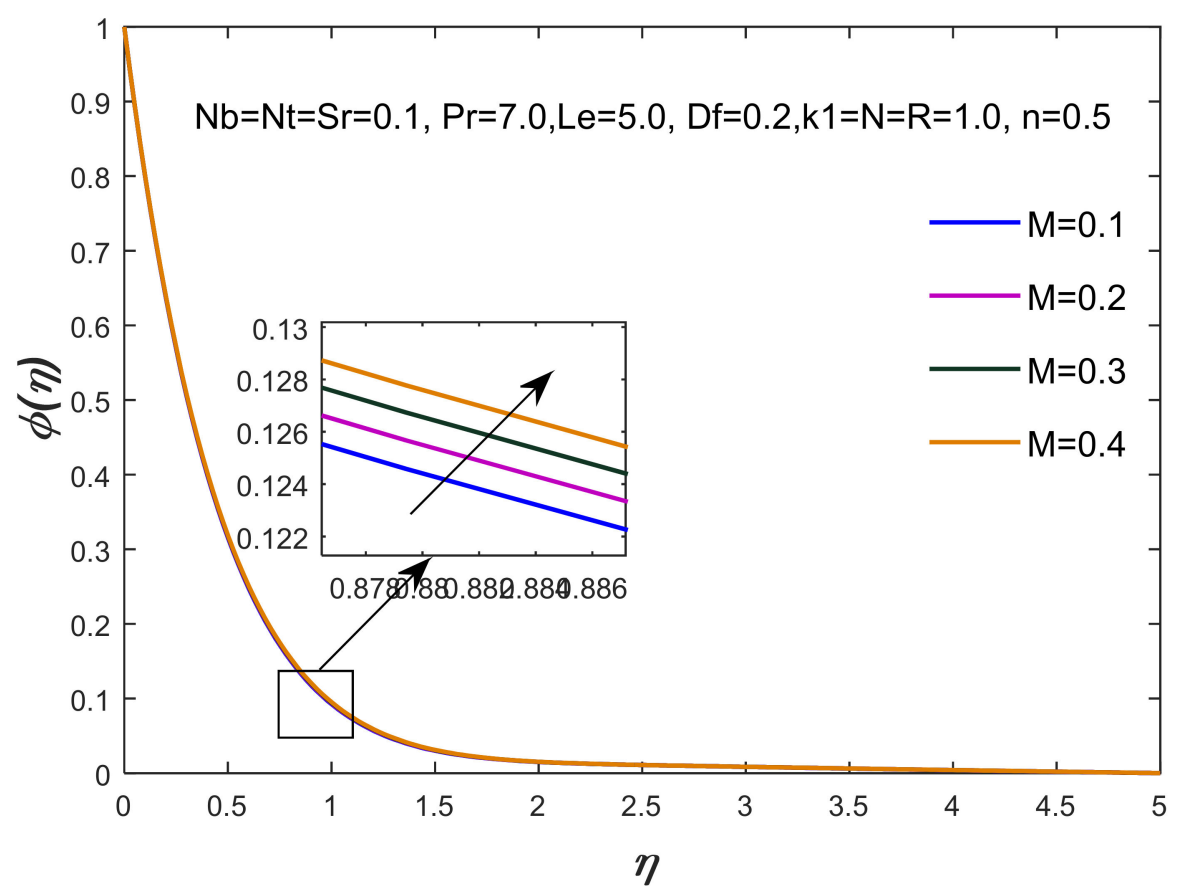

Figure 16. The impacts of $M$ on $\phi(\eta)$.

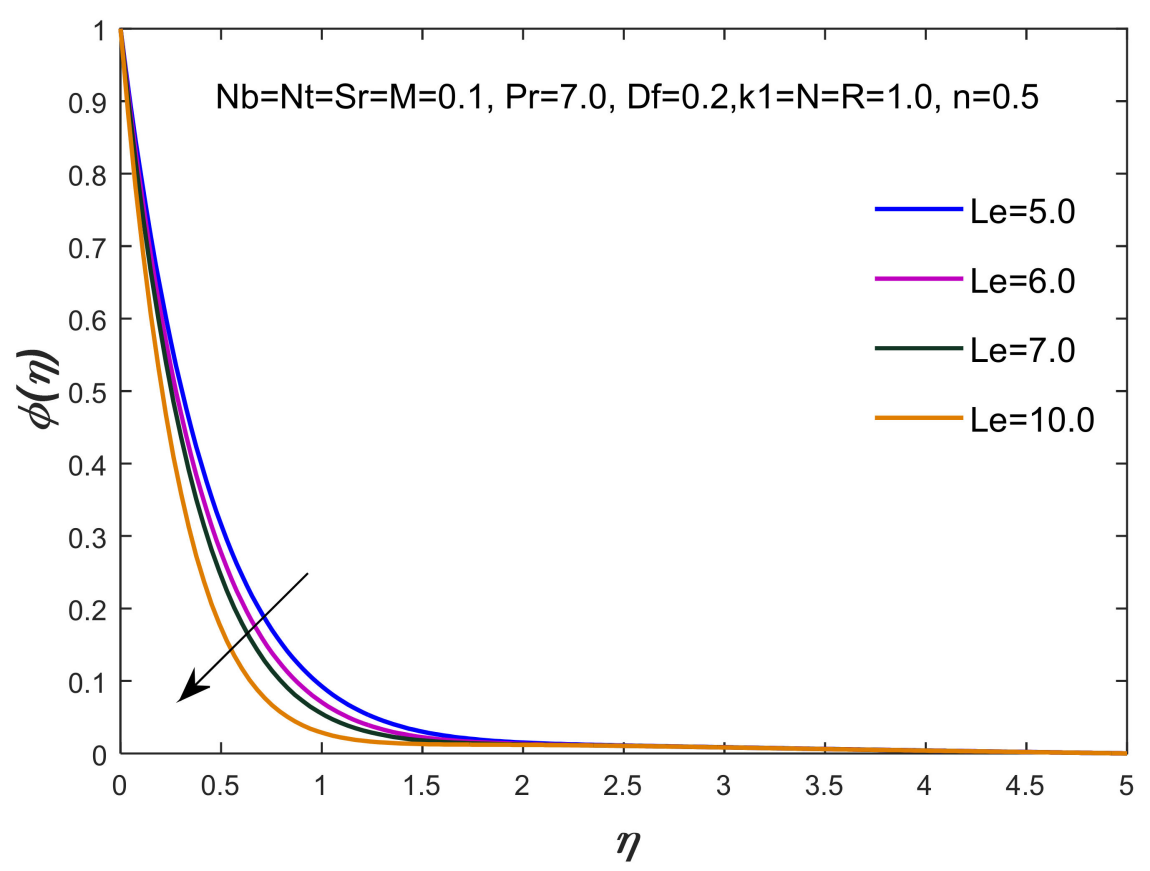

Figure 17. The impacts of Le on $\phi(\eta)$. 


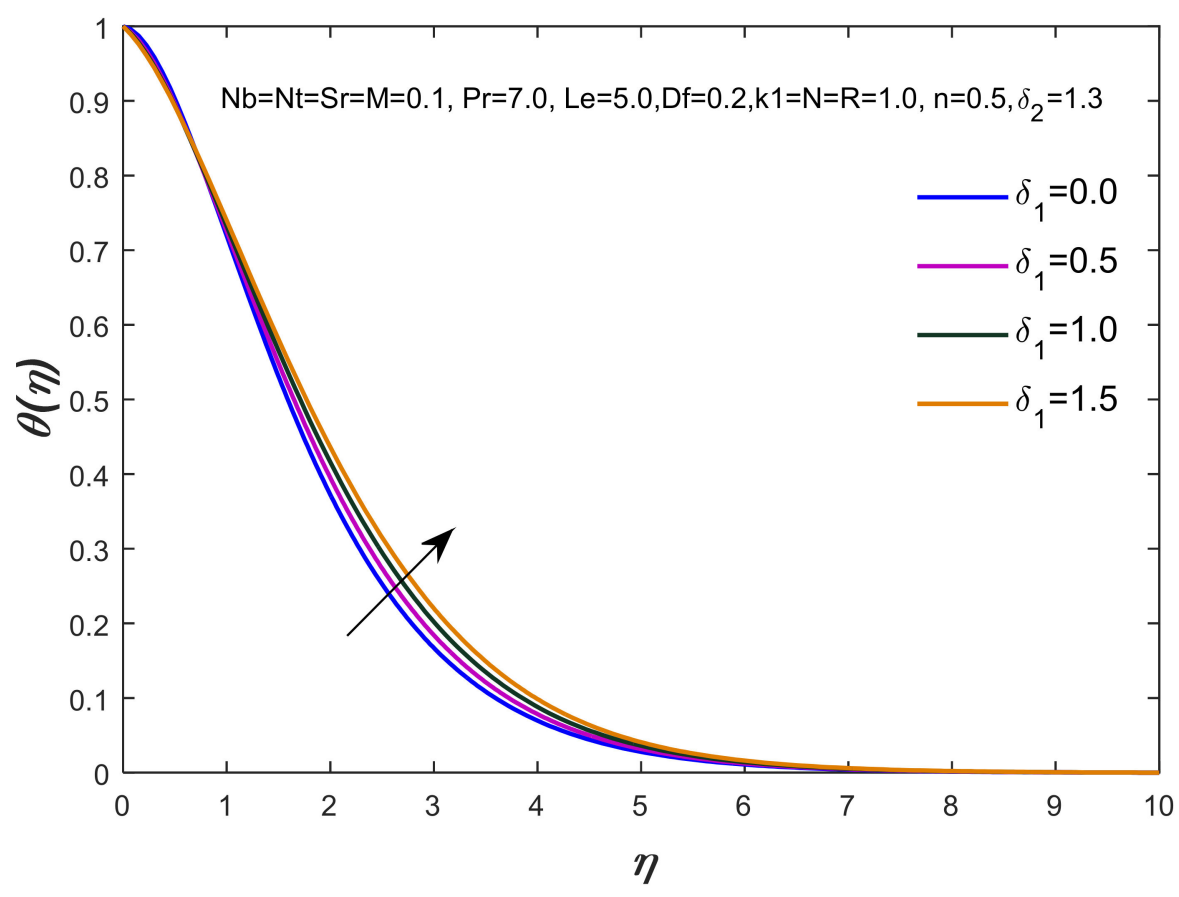

Figure 18. The impacts of $\delta_{1}$ on $\phi(\eta)$.

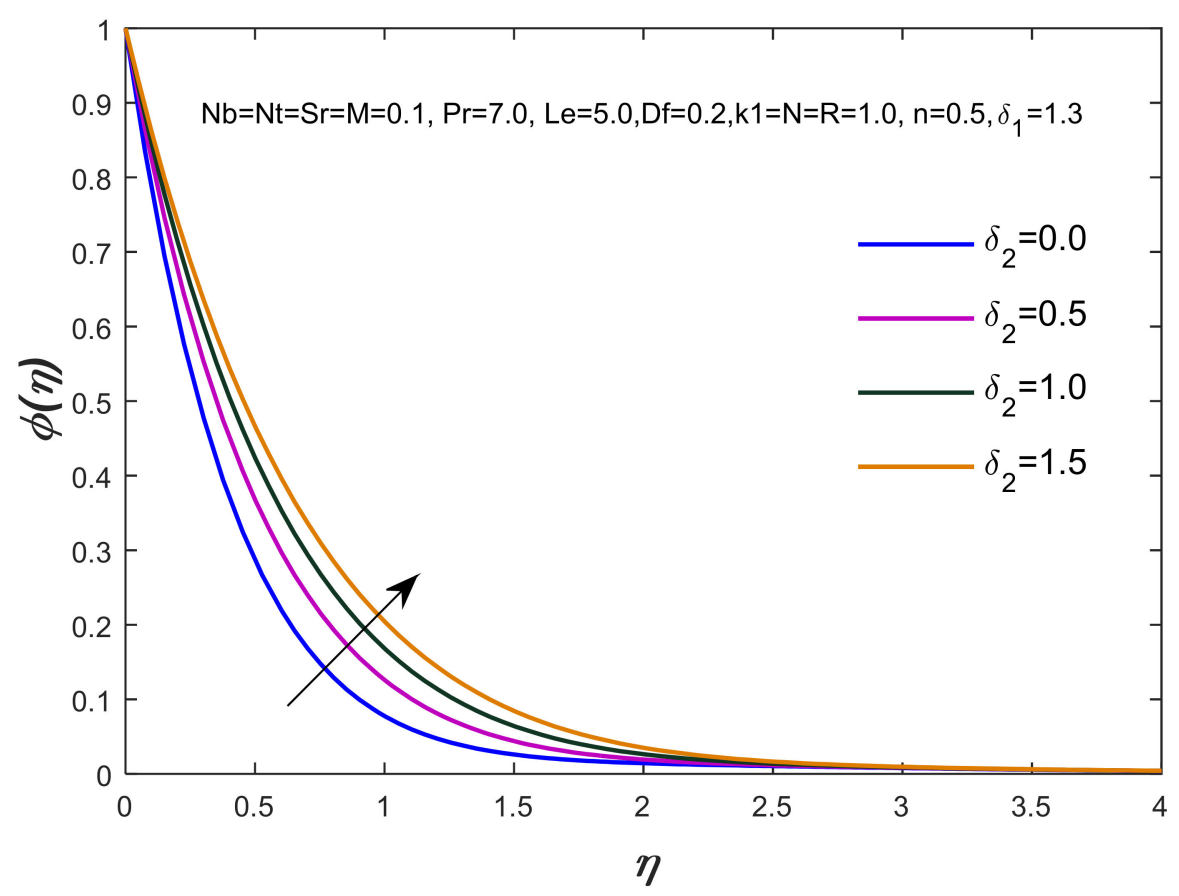

Figure 19. The impacts of $\delta_{2}$ on $\phi(\eta)$.

Figure 14 reveals the variation of $(\mathrm{Nb})$ subjected to particles concentration whereas a reduction in the curves of concentration is found by using large values of $\mathrm{Nb}$. Moreover, the Brownian motion number and the Lewis number are treated opposite to each other.

An enhancement in $(N t)$ generates better performance of heat energy and shear gradient (see Figure 15). The increasing function of thermal energy is found versus $(N t)$. Meanwhile, fluid particles are experience more heat energy versus large values of $N t$.

The action of $(M)$ on the temperature curves are established by Figure 16. From this figure, the diffusion of nanoparticles is enhanced through variation in the magnetic field. This impact is due to the Lorentz force because this force generates a reduction in the 
momentum boundary. Hence, the concentration boundary layer is reduced, therefore, a better performance regarding diffusion of nanoparticles at the wall of the surface.

Figure 17 captures the diffusion of nanoparticles against increasing values of the Lewis number $(L e)$. It is noticed that concentration curves under the action of the Lewis number is increased. Moreover, a reduction in the concentration boundary layer is investigated versus large values of $(L e)$.

The dimensionless temperature profile under the variation of thermal relaxation time is conducted in Figure 18. From this figure, graphical outcomes capture that the fluid temperature enhances when thermal relaxation time $\left(\delta_{1}\right)$ is enhanced. Meanwhile, the TBL increases.

The increasing function associated with the concentration curves versus the growing values of concentration relation time number $\left(\delta_{2}\right)$ is simulated by Figure 19. It reveals that the diffusion of nanoparticles becomes fast.

Divergent force, Nusselt number and rate of diffusion of fluid particles versus variation of physical parameters: The comparative study is presented in Table 1 . The numerical simulations in view of the divergent velocity, rate of thermal energy, and diffusion of mass versus physical parameters called the Brownian motion $(\mathrm{Nb})$, thermophoresis $(N t)$,Prandtl number $(P r)$, Lewis number $(L e)$, magnetic field $(M)$, porosity number $(k 1)$, thermal radiation $(R)$, Soret number $(S r)$, Dufour number $(D r)$, stratification number $(n)$, concentration relaxation time $\left(\delta_{2}\right)$, and thermal relaxation time $\left(\delta_{1}\right)$ are recorded in Table 2. It is observed from this table that divergent velocity is enhanced through variation of $(P r)$ becaus $(P r)$ is based on the variation of thermal layer as well as the momentum layer at the wall of the surface. The opposite character in view of divergent velocity is noted versus increasing values of the magnetic field and porosity numbers. The divergent velocity becomes slower versus the variation of the thermal radiation number $(R)$. Furthermore, it can be observed that constant values regarding shear stress are recorded using the values of $(N b),(N t),(L e),(S r),(n),\left(\delta_{2}\right)$, and $\left(\delta_{1}\right)$. The rate of diffusion of nanoparticles becomes faster versus $N b, N t, L e$, and $k 1$. However, reduction in view of the Sherwood number is simulated under the variation of the Prandtl number, magnetic field, thermal relaxation time, concentration relaxation time, and the Soret and Dufour numbers. The maximum performances related to thermal energy are observed against growing values of $N b, N t, P r, M, k 1, \delta_{1}$, and $\delta_{2}$. The increase in the Nusselt number is computed versus $L e, \Gamma, D r, S r$, and $n$. The comparative study is presented in Table 1 for the heat transfer rate. It is obvious that the obtained results are in excellent agreement with the findings reported in reference [19].

Table 1. The value of $-\theta \prime(0)$ for $N t=N b=k 1=D f=S r=L e=n=\Gamma=0$.

\begin{tabular}{ccccc}
\hline $\boldsymbol{P r}$ & $\boldsymbol{M}$ & $\boldsymbol{R}$ & $\begin{array}{c}-\boldsymbol{\theta}^{\prime}(\mathbf{0}) \\
{[\mathbf{1 9 ]}}\end{array}$ & $\begin{array}{c}-\boldsymbol{\theta}^{\prime}(\mathbf{0}) \\
\text { Present Results }\end{array}$ \\
\hline 1.0 & 0.0 & 0.0 & 0.9548 & 0.95484878 \\
2.0 & 0.0 & 0.0 & 1.4714 & 1.4714548 \\
3.0 & 0.0 & 0.0 & 1.8691 & 1.86906988 \\
1.0 & 0.0 & 1.0 & 0.5315 & 0.53530132 \\
1.0 & 1.0 & 0.0 & 0.8611 & 0.86150565 \\
1.0 & 1.0 & 1.0 & 0.4505 & 0.46196426 \\
\hline
\end{tabular}


Table 2. Calculation of $-\theta \prime(0),-\varnothing \prime(0)$ and $f^{\prime \prime}(0)$ with variation of thermophysical parameters.

\begin{tabular}{cccccccccccccccc}
\hline $\boldsymbol{N b}$ & $\boldsymbol{N} \boldsymbol{t}$ & $\boldsymbol{P r}$ & $\boldsymbol{L} \boldsymbol{e}$ & $\boldsymbol{M}$ & $\boldsymbol{k} \mathbf{1}$ & $\boldsymbol{R}$ & $\boldsymbol{\Gamma}$ & $\boldsymbol{D f}$ & $\boldsymbol{S r}$ & $\boldsymbol{n}$ & $\boldsymbol{\delta} \mathbf{1}$ & $\boldsymbol{\delta} \mathbf{2}$ & $-\boldsymbol{f}^{\prime \prime}(\mathbf{0})$ & $-\boldsymbol{\theta}^{\prime}(\mathbf{0})$ & $-\boldsymbol{\emptyset}^{\prime}(\mathbf{0})$ \\
\hline 0.1 & 0.1 & 7.0 & 5.0 & 0.1 & 0.1 & 0.1 & 0.1 & 0.1 & 0.1 & 0.1 & 1.3 & 1.3 & 1.358958 & 1.203130 & 1.692398 \\
0.5 & 0.1 & 7.0 & 5.0 & 0.1 & 0.1 & 0.1 & 0.1 & 0.1 & 0.1 & 0.1 & 1.3 & 1.3 & 1.358958 & 0.745205 & 1.730309 \\
0.1 & 0.3 & 7.0 & 5.0 & 0.1 & 0.1 & 0.1 & 0.1 & 0.1 & 0.1 & 0.1 & 1.3 & 1.3 & 1.358958 & 0.993797 & 1.705696 \\
0.1 & 0.1 & 8.0 & 5.0 & 0.1 & 0.1 & 0.1 & 0.1 & 0.1 & 0.1 & 0.1 & 1.3 & 1.3 & 1.358958 & 1.260018 & 1.691140 \\
0.1 & 0.1 & 7.0 & 10.0 & 0.1 & 0.1 & 0.1 & 0.1 & 0.1 & 0.1 & 0.1 & 1.3 & 1.3 & 1.358958 & 1.020337 & 2.459589 \\
0.1 & 0.1 & 7.0 & 5.0 & 2.5 & 0.1 & 0.1 & 0.1 & 0.1 & 0.1 & 0.1 & 1.3 & 1.3 & 2.063846 & 1.080128 & 1.514741 \\
0.1 & 0.1 & 7.0 & 5.0 & 0.1 & 2.0 & 0.1 & 0.1 & 0.1 & 0.1 & 0.1 & 1.3 & 1.3 & 1.93865 & 1.103093 & 1.545366 \\
0.1 & 0.1 & 7.0 & 5.0 & 0.1 & 0.1 & 3.0 & 0.1 & 0.1 & 0.1 & 0.1 & 1.3 & 1.3 & 1.358958 & 0.628864 & 3.137834 \\
0.1 & 0.1 & 7.0 & 5.0 & 0.1 & 0.1 & 0.1 & 5.0 & 0.1 & 0.1 & 0.1 & 1.3 & 1.3 & 1.358958 & 0.458712 & 1.664456 \\
0.1 & 0.1 & 7.0 & 5.0 & 0.1 & 0.1 & 0.1 & 0.1 & 0.3 & 0.1 & 0.1 & 1.3 & 1.3 & 1.358958 & 0.688129 & 1.720053 \\
0.1 & 0.1 & 7.0 & 5.0 & 0.1 & 0.1 & 0.1 & 0.1 & 0.1 & 0.3 & 0.1 & 1.3 & 1.3 & 1.358958 & 1.287439 & 1.347656 \\
0.1 & 0.1 & 7.0 & 5.0 & 0.1 & 0.1 & 0.1 & 0.1 & 0.1 & 0.1 & 0.2 & 1.3 & 1.3 & 1.358958 & 1.045916 & 1.696398 \\
0.1 & 0.1 & 7.0 & 5.0 & 0.1 & 0.1 & 0.1 & 0.1 & 0.1 & 0.1 & 0.1 & 1.5 & 1.3 & 1.358958 & 1.179728 & 1.694022 \\
0.1 & 0.1 & 7.0 & 5.0 & 0.1 & 0.1 & 0.1 & 0.1 & 0.1 & 0.1 & 0.1 & 1.3 & 1.5 & 1.358958 & 1.216942 & 1.635957 \\
\hline
\end{tabular}

\section{Conclusions}

The development of a 2D model in the presence of momentum, heat energy, and concentration equations is designed. The influence the variation of the magnetic field and the Dufour and Soret numbers has on the Newtonian rheological fluid passing over a porous hot surface is observed. This developed model is numerically solved using a shooting approach. The key simulations are conducted as follows:

- The flow of nanoparticles on a hot surface is managed through the variation of magnetic field and porosity numbers. It is simulated that the motion of nanoparticles becomes slower versus growing values of the magnetic field and porosity numbers. The thickness of the MBL is investigated in view of this reduction;

- The intensity of magnetic field plays a vital role on heat energy and the TBL because thermal performance has improved under the action of a magnetic field. Moreover, the transportation of temperature becomes slowr versus an increment in the Prandtl number called ration of MBL to TBL;

- The maximum production of thermal energy has been observed with respect to the variation of the thermal radiation, thermophoresis, Brownian motion and $\mathrm{Du}-$ four numbers, whereas thickness of the TBL declines by changing the values of the Dufour number;

- The diffusion of fluid particles has been observed significantly against the variation of thermophoresis, Brownian motion, magnetic field, thermal relaxation time and concentration relaxation time numbers;

- The divergent velocity speeds up versus the Prandtl number, magnetic field, and the thermal radiation numbers, but reduces in speed in view of divergent flow against large values of the thermal radiation number.

Author Contributions: Data curation, M.S., U.N. and T.N.; Formal analysis, M.S. and U.N.; Funding acquisition, U.N. and A.M.A.; Investigation, M.S., U.N. and E.R.E.-Z.; Methodology, M.S., U.N., E.R.E.-Z. and T.N.; Project administration, M.S. and A.M.A.; Resources, M.S., U.N. and E.R.E.-Z.; Software, M.S., U.N., E.R.E.-Z. and A.M.A.; Supervision, M.S.; Validation, M.S., U.N. and E.R.E.-Z.; Visualization, M.S. and U.N.; Writing—original draft, M.S., U.N., E.R.E.-Z. and A.M.A.; Writingreview \& editing, M.S., U.N. and E.R.E.-Z. All authors have read and agreed to the published version of the manuscript.

Funding: This research received no external funding.

Institutional Review Board Statement: Not applicable.

Informed Consent Statement: Not applicable.

Data Availability Statement: The data used to perform this research is included in the manuscript. 
Acknowledgments: The authors acknowledge that this publication was supported by the Dean ship of Scientific Research at Prince Sattam bin Abdulaziz University, Alkharj, Saudi Arabia.

Conflicts of Interest: The authors declare that they have no conflicts of interest to report regarding the present study.

\section{Nomenclature}

$\begin{array}{ll}u, v & \text { Velocity field } \\ T & \text { Temperature } \\ \rho & \text { Fluid density } \\ \delta_{1}, \delta_{2} & \text { Small parameters } \\ \theta(\eta) & \text { Dimensionless temperature } \\ k 1 & \text { Porosity parameter } \\ L e & \text { Lewis number } \\ -\phi \prime(0) & \text { Mass transfer rate } \\ R & \text { Radiation parameter } \\ \psi & \text { Stream function } \\ D(T) & \text { Temperature dependent diffusion coefficient } \\ C_{w} / C_{\infty} & \text { Wall concentration/ambient concentration } \\ x, y & \text { Space coordinates } \\ C & \text { Concentration } \\ \Gamma & \text { Chemical reaction parameter } \\ M & \text { Magnetic parameter } \\ \phi(\eta) & \text { Dimensionless concentration } \\ f(\eta), f \prime(\eta) & \text { Dimensionless velocity } \\ \Gamma & \text { Chemical reaction parameter } \\ -(1+R) \theta^{\prime}(0) & \text { Heat transfer rate } \\ K(T) & \text { Temperature dependent thermal conductivity } \\ \eta & \text { Dimensionless independent variable } \\ T_{w} / T_{\infty} & \text { Wall temperature/ambient temperature } \\ \tau_{W}=\mu \frac{\partial u}{\partial y} & \text { Wall stress } \\ & \end{array}$

\section{References}

1. Sohail, M.; Naz, R.; Bilal, S. Thermal performance of an MHD radiative Oldroyd-B nanofluid by utilizing generalized models for heat and mass fluxes in the presence of bioconvective gyrotactic microorganisms and variable thermal conductivity. Heat Transfer Asian Res. 2019, 48, 2659-2675. [CrossRef]

2. Khan, M.I.; Kiyani, M.Z.; Malik, M.Y.; Yasmeen, T.; Khan, M.W.A.; Abbas, T. Numerical investigation of magnetohydrodynamic stagnation point flow with variable properties. Alex. Eng. J. 2016, 55, 2367-2373. [CrossRef]

3. Sohail, M.; Ali, U.; Al-Mdallal, Q.; Thounthong, P.; Sherif, E.S.M.; Alrabaiah, H.; Abdelmalek, Z. Theoretical and numerical investigation of entropy for the variable thermophysical characteristics of couple stress material: Applications to optimization. Alex. Eng. J. 2020, 59, 4365-4375. [CrossRef]

4. Khan, M.I.; Waqas, M.; Hayat, T.; Alsaedi, A. Chemically reactive flow of micropolar fluid accounting viscous dissipation and Joule heating. Results Phys. 2017, 7, 3706-3715. [CrossRef]

5. Hayat, T.; Ahmad, S.; Khan, M.I.; Alsaedi, A. A frame work for heat generation/absorption and modified homogeneousheterogeneous reaction in flow based on non-Darcy-Forchheimer medium. Nucl. Eng. Technol. 2018, 50, 389-395. [CrossRef]

6. Sohail, M.; Shah, Z.; Tassaddiq, A.; Kumam, P.; Roy, P. Entropy generation in MHD Casson fluid flow with variable heat conductance and thermal conductivity over non-linear bi-directional stretching surface. Sci. Rep. 2020, 10, 1-16. [CrossRef] [PubMed]

7. Shah, Z.; Sheikholeslami, M.; Kumam, P.; Shafee, A. Modeling of entropy optimization for hybrid nanofluid MHD flow through a porous annulus involving variation of Bejan number. Sci. Rep. 2020, 10, 1-14. [CrossRef] [PubMed]

8. Shah, Z.; McCash, L.B.; Dawar, A.; Bonyah, E. Entropy optimization in Darcy-Forchheimer MHD flow of water based copper and silver nanofluids with Joule heating and viscous dissipation effects. AIP Adv. 2020, 10, 065137. [CrossRef]

9. Khan, U.; Shafiq, A.; Zaib, A.; Sherif, E.S.M.; Baleanu, D. MHD Radiative Blood Flow Embracing Gold Particles via a Slippery Sheet through an Erratic Heat Sink/Source. Mathematics 2020, 8, 1597. [CrossRef]

10. Rasool, G.; Shafiq, A. Numerical exploration of the features of thermally enhanced chemically reactive radiative Powell-Eyring nanofluid flow via Darcy medium over non-linearly stretching surface affected by a transverse magnetic field and convective boundary conditions. Appl. Nanosci. 2020, 53, 1-18.

11. Al-Khaled, K.; Khan, S.U. Thermal Aspects of Casson Nanoliquid with Gyrotactic Microorganisms, Temperature-Dependent Viscosity, and Variable Thermal Conductivity: Bio-Technology and Thermal Applications. Inventions 2020, 5, 39. [CrossRef] 
12. Mandal, I.C.; Mukhopadhyay, S. Heat transfer analysis for fluid flow over an exponentially stretching porous sheet with surface heat flux in porous medium. Ain Shams Eng. J. 2013, 4, 103-110. [CrossRef]

13. Khan, S.U.; Ali, N.; Abbas, Z. Hydromagnetic flow and heat transfer over a porous oscillating stretching surface in a viscoelastic fluid with porous medium. PLOS ONE 2015, 10, e0144299.

14. Alam, A.; Marwat, D.N.K. Heat and mass transfer on a stretching/shrinking and porous sheet of variable thickness with suction and injection. Proc. Inst. Mech. Eng. Part C J. Mech. Eng. Sci. 2021, 0954406220982333. [CrossRef]

15. Abdelsalam, S.I.; Sohail, M. Numerical approach of variable thermophysical features of dissipated viscous nanofluid comprising gyrotactic micro-organisms. Pramana J. Phys. 2020, 94, 1-12. [CrossRef]

16. Sohail, M.; Naz, R.; Shah, Z.; Kumam, P.; Thounthong, P. Exploration of temperature dependent thermophysical characteristics of yield exhibiting non-Newtonian fluid flow under gyrotactic microorganisms. AIP Adv. 2019, 9, 125016. [CrossRef]

17. EL-Kabeir, S.M.; El-Zahar, E.R.; Rashad, A.M. Effect of chemical reaction on heat and mass transfer by mixed convection flow of casson fluid about a sphere with partial slip. J. Comput. Theor. Nanosci. 2016, 13, 5218-5226. [CrossRef]

18. Sohail, M.; Naz, R. Modified heat and mass transmission models in the magnetohydrodynamic flow of Sutterby nanofluid in stretching cylinder. Phys. A Stat. Mech. Appl. 2020, 549, 124088. [CrossRef]

19. Anwar, M.I.; Ali, M.; Rafique, K.; Shehzad, S.A. Soret-Dufour and radiative aspects in hydromagnetized nanofluid flow in stratified porous medium. SN Appl. Sci. 2019, 1, 1430. [CrossRef] 